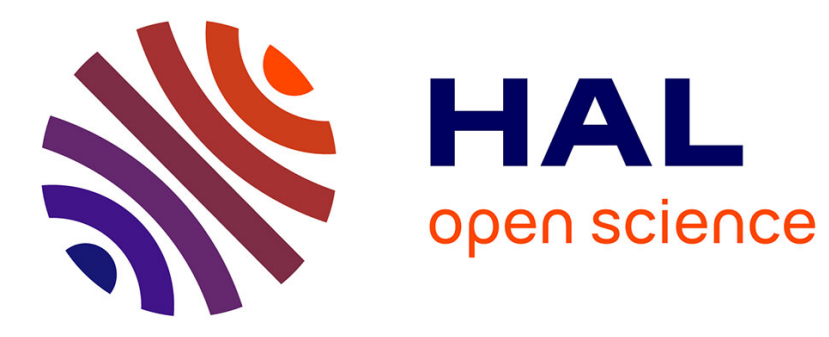

\title{
Survey on Traffic of Metro Area Network with Measurement On-Line
}

Gaogang Xie, Guangxing Zhang, Jianhua Yang, Yinghua Min, Valérie Issarny, Alberto Conte

\section{- To cite this version:}

Gaogang Xie, Guangxing Zhang, Jianhua Yang, Yinghua Min, Valérie Issarny, et al.. Survey on Traffic of Metro Area Network with Measurement On-Line. International Teletraffic Congress, 2007, Ottawa, Canada. pp.666-677. inria-00415923

\section{HAL Id: inria-00415923 https://hal.inria.fr/inria-00415923}

Submitted on 11 Sep 2009

HAL is a multi-disciplinary open access archive for the deposit and dissemination of scientific research documents, whether they are published or not. The documents may come from teaching and research institutions in France or abroad, or from public or private research centers.
L'archive ouverte pluridisciplinaire HAL, est destinée au dépôt et à la diffusion de documents scientifiques de niveau recherche, publiés ou non, émanant des établissements d'enseignement et de recherche français ou étrangers, des laboratoires publics ou privés. 


\title{
Survey on Traffic of Metro Area Network with Measurement On-Line
}

\author{
Gaogang Xie ${ }^{1,2,4}$, Guangxing Zhang ${ }^{1,3}$, Jianhua Yang ${ }^{1}$, Yinghua Min ${ }^{1}$, \\ Valerie Issarny $^{2}$, and Alberto Conte ${ }^{4}$ \\ ${ }^{1}$ Institute of Computing Technology, Chinese Academy of Sciences, \\ Beijing, 100080, P.R. China \\ ${ }^{2}$ INRIA-Rocquencourt, Domaine de Voluceau, 78153 Le Chesnay, France \\ ${ }^{3}$ Computer and Communication School, Hunan University, ChangSha, 410082, P.R. China \\ ${ }^{4}$ Alcatel Research \& Innovation Center, Marcoussis, France \\ \{xie, guangxing, jhyang, min\}@ict.ac.cn, \\ Valerie.issarny@inria.fr, alberto.contedalcatel.fr
}

\begin{abstract}
Network traffic measurements can provide essential data for network research and operation. While Internet traffic has been heavily studied for several years, there are new characteristics of traffic having not been understood well brought by new applications for example P2P. It is difficult to get these traffic metrics due to the difficulty to measurement traffic on line for high speed link and to identify new applications using dynamic ports. In this paper, we present a broad overview of Internet traffic of an operated OC-48 export link of a metro area network from a carrier with the method of measurement on-line. The traffic behaves a daily characteristic well and the traffic data of whole day from data link layer to application layer is presented. We find the characteristics of traffic have changed greatly from previous measurements. Also, we explain the reasons bringing out these changes. Our goal is to provide the first hand of traffic data that is helpful for people to understand the change of traffic with new applications.
\end{abstract}

Keywords: Internet Traffic, Metro Area Network, Measurement.

\section{Introduction}

Traffic metrics not only provide insight into the design, operation and usage patterns of operated network $[1,2,3,4]$, but also are used in network research and device development [5, 6]. Since Ramon Caceres captured the first published measurements of a size's wide area Internet traffic in 1989 [7], great effort and progress have been made in the research area of traffic measurement [8-13]. For instance, the characterization of self-similar nature of traffic is revealed from traffic measurement [4]. New applications, for example P2P-based VoIP, have not only changed characteristics of traffic and behavior of network, but also proposed more different requirements on QoS provisioning ability of network infrastructure. To understand the behavior of network and applications, firstly we should investigate the traffic characteristic of different applications. But, it is far away enough to just measure the 
traffic rate at data link layer in order to reveal how the characterizations of traffic to form. More metrics such as traffic proportion at application layer, concurrent flows and packets dynamic with different priorities, are necessary for network planning, operating and research to meet QoS requirement of every application.

Although Pang and others [9] look at enterprise traffic , P2P traffic is not included in their networks under test. In fact, P2P traffic dominates the traffic in other enterprise networks [14]. In addition, enterprise network is often affected by a few hosts. Some statistic characteristics is then not so obvious. Metro area network (MAN) as PoPs of Internet connecting millions of users into backbone plays key role in Internet. It affects the performance of application directly. Most complicated traffic engineering policies and operating rules are also deployed in MAN. Thus, it is important to characterize traffic of MANs.

On the other hand, the difficulty in characterizing MAN traffic comes from high speed of links [15] and new application identifications [16]. It is very difficult with the most powerful hardware to map every packet into the list of millions flows using the methodology with the time complexity $O(l)$ in line speed to measure the flowlevel metrics where $l$ is the count of concurrent flows, for up to OC-192/OC-768 backbone link [15]. On the other hand, the determinate ports in the headers of packets are used to identify applications associated with a particular port and thus of a particular application before. It is well known that such a process is likely to lead to inaccurate estimates of the amount of traffic carried by different applications given that specific protocol $[14,16]$. For example, some P2P applications tends to use dynamic protocol ports for not being blocked by firewall or bandwidth management device, even tunneled through HTTP with 80 protocol port [16]. These are likely reasons why MAN traffic has not been studied extensively.

This paper explores the traffic characteristics from 5 months measurement experimentations on an export link of a carrier's operating MAN. Thanks to the accurate MPI traffic identification technology in our measurement infrastructure NetTurbo $[14,16]$, we get a large amount of data to characterize traffic from packetlevel to flow-level, from data link layer traffic to application layer traffic. To the best of our knowledge, this is the first paper giving the overview of the characteristics of MAN traffic based on long term on line measurement. Since the traffics behave obviously daily characteristic, only the traffics in one day is analyzed. The main goal of our research is to provide a broad overview of traffic characteristic of MAN.

- The traffic is analyzed according to inbound, outbound and bidirectional.

- The traffic breakdown is made from data link layer to application layer with our accurate traffic identification method MPI.

- The traffic metrics of packet level, byte level and flow level are listed.

- P2P and VoIP traffic rates are presented.

- Traffic rate and traffic amount are presented.

- The traffic of OC-48 link in one whole day is analyzed instead of several minutes trace.

The rest of this paper is organized as follows. Section 2 describes our measurement experiment and the traffic dataset. In Section 3, the makeup of traffic from data link layer to application layer is discussed. We also introduce the flows and IP pairs in this 
section. In Section 4, the relative work is presented. Finally, we conclude and discuss our future work in Section 5.

\section{Measurement Experiment and Dataset}

A traffic measurement system named NetTurbo supporting traffic monitoring from data link layer to application layer, which is developed by ourselves and is composed several measurement probes named NetPro and a GUI based data collection and analysis software named NetCom, is deployed in a carrier's metro area network as fig. 1. The measurement probe has been installed in the OC-48 POS export link through an optical splitter and monitored the bidirectional traffics since May 182006. All traffic metrics in this paper are produced in NetPro, pushed to NetCom every 10 seconds through management network port, and stored into the database server for history analysis. Our traffic identification method named MPI [16] is used in NetPro to identify new appearing applications such as VoIP and $\mathrm{P} 2 \mathrm{P}$ in order to understand the makeup of traffic in application level. The link under tested is one of two export links of a metro area network of a main carrier in China connected to the national backbone between two Cisco's GSR 12416 routers. The population is about 3.8 million living in the region, and among them 1.4 million in the city and the others in the countryside. All of accessing the resource of Internet outside the city is transmitted through the two export links.

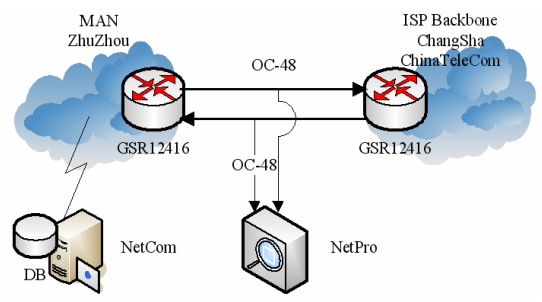

Fig. 1. Measure Experiment

The measurement experiment is performed in two phases. The first phase is from May 18 to September 20 and the second phase is from October 8 to November 1 . The main difference in the two data sets is more application protocols identified in the second phases with an improvement over our MPI. So, only the data set of the second phase is analyzed in this paper.

\section{Traffic Breakdown at Different Layers}

\subsection{Traffic Overview}

We select traffic in a week randomly and the traffic data present obviously daily characteristic as fig. 2 . 


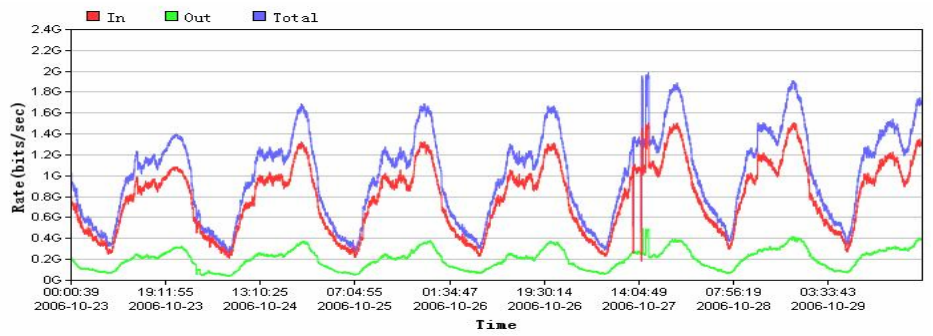

Fig. 2. Traffic Rate in a Week

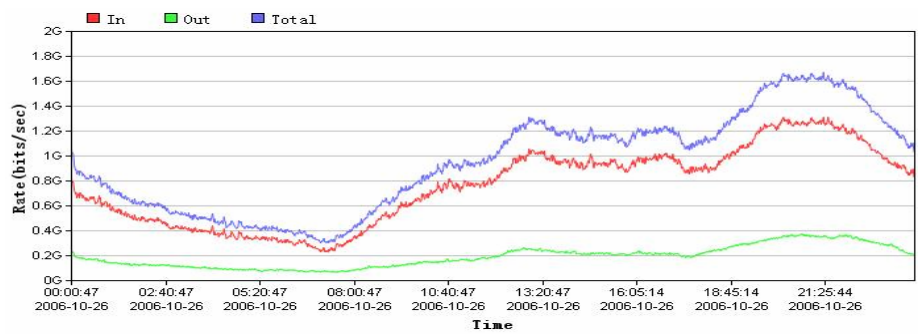

Fig. 3. Traffic Rate on Oct. 262006

The maximum traffic rate is $1507 \mathrm{Mbps}$ for inbound direction, $499.1 \mathrm{Mbps}$ for outbound direction and $1987 \mathrm{Mbps}$ in total of bidirection, those of the minimum traffic rates are $181.6 \mathrm{Mbps}, 41.8 \mathrm{Mbps}$ and $260.1 \mathrm{Mbps}$ and those of average traffic rates are $808.4 \mathrm{Mbps}, 205.6 \mathrm{Mbps}$ and $1014 \mathrm{Mbps}$ in the week. The peak traffic rate appears from 9:00 pm 10:00 pm usually and the bottom appears from 6:30am to 7:30 am every day. The traffic burst in Fig.2 in October 27 is caused by the mechanism of traffic load balance between two export links. Since there is the characteristic of day, the traffic from on October 26 is selected for advanced analysis as fig. 3. The overview of traffic rate of the traffic is shown in table 1.

Table 1. Summary of Traffic Rate on October 26

\begin{tabular}{|c|c|c|c|c|c|c|}
\hline & \multicolumn{3}{|c|}{ BPS } & \multicolumn{3}{c|}{ pps } \\
\cline { 2 - 7 } & Max & Min & Avg & Max & Min & Avg \\
\hline Inbound & 1311 & 230.7 & 760.9 & 297520 & 49267 & 167111 \\
\hline Outbound & 374 & 68.27 & 192.1 & 127765 & 21558 & 68441 \\
\hline Bidirection & 1671 & 304.3 & 953 & 424043 & 72211 & 235552 \\
\hline
\end{tabular}

About $19.17 \%$ of packets, i.e. about $1.76 \%$ of bytes, are with 64 byte in a packet, whereas about $16.32 \%$ of packets, i.e. $42.90 \%$ in bytes are with from 1024 to 1518 bytes in a packet. The characteristic of tri-modal for packet size distribution in our measurement is not as obvious as previous research [19, 20]. Thompson et al. [19] developed a high performance monitoring system with OC-3 interface capturing 
traffic and performed measurement experiments on the OC-3 trunks within internet MCI's backbone and also within the NSF-sponsored vBNS. They reported on measurements from two OC-3 trunks in the presence of up to 240000 flows. The Packet size distribution is tri-modal in their result: $40-44$ bytes $(20 \%),<=552$ or 576 bytes $(65 \%)$ and 1500 bytes $(15 \%)$. McReary [20] also reported the similar result from the NASA Ames Internet Exchange over 10 months in 2000. The average packet size for every application category is shown as Table 2.c. New applications such as P2P and VoIP change the packet size distribution. It is easily to be understood for HTTP the average packet size 959.87 bytes for inbound link and 155.71 bytes for outbound due to more big packets for data in inbound link and 64 byte packets for acknowledgement in outbound link. It is also well to be understood the packet sizes are similar in inbound link and outbound link for P2P applications. But, it is unknown why the packet size in inbound link is larger than that in outbound link for instant messenger and VoIP.

Table 2. Distribution of Packet Size

(A) IN BYTE AND PACKETS $(\%)$

\begin{tabular}{|l|c|c|c|c|c|c|}
\hline \multirow{2}{*}{ Pkt_size } & \multicolumn{3}{|c|}{ In Bytes (\%) } & \multicolumn{3}{c|}{ In Packets (\%) } \\
\cline { 2 - 7 } & Inbound & Outbound & Bidirection & Inbound & Outbound & Bidirection \\
\hline $0 \sim 64$ & 1.76 & 6.21 & 2.66 & 19.17 & 42.54 & 25.96 \\
\hline $64 \sim 128$ & 3.25 & 5.01 & 3.61 & 23.10 & 22.03 & 22.79 \\
\hline $128 \sim 256$ & 5.59 & 6.31 & 5.73 & 15.83 & 11.14 & 14.47 \\
\hline $256 \sim 512$ & 10.11 & 10.07 & 10.10 & 8.90 & 5.38 & 7.88 \\
\hline $512 \sim 1024$ & 36.39 & 39.52 & 37.02 & 16.68 & 11.17 & 15.08 \\
\hline $1024 \sim 1518$ & 42.90 & 32.88 & 40.88 & 16.32 & 7.74 & 13.83 \\
\hline$>1518$ & 0.00 & 0.00 & 0.00 & 0.00 & 0.00 & 0.00 \\
\hline
\end{tabular}

(B) PACKET SIZE FOR EVERY APPLICATION CATEGORY (BYTES)

\begin{tabular}{|l|l|l|l|}
\hline Apps & Inbound & Outbound & Bidirection \\
\hline Protocols in RFCs & 959.87 & 155.71 & 756.43 \\
\hline P2P File Sharing & 779.42 & 616.76 & 724.37 \\
\hline Instant Messenger & 359.89 & 131.83 & 285.07 \\
\hline Games & 220.47 & 203.54 & 217.30 \\
\hline Streaming Media & 791.42 & 309.70 & 643.95 \\
\hline VoIP & 241.02 & 193.35 & 232.60 \\
\hline Uncategorized & 322.24 & 275.55 & 307.84 \\
\hline
\end{tabular}

In network layer, almost all the traffic is IPv4 and the summation of other protocols traffic such as PPP, GSMP and others is less than $0.001 \%$. As to transport layer, the traffic of TCP is still dominating over UDP both in inbound and outbound, and both in packets and in bytes as Table 3. There are $95 \%$ of bytes and $90 \%$ packets for TCP and 5\% bytes, 10\% packets for UDP in Thompson's experiment in 1997 [19]. McReary [20] in 2000 and Fraleigh [22] in 2003 also got the similar results with Thompson. The reason why UDP traffic increases is more applications such VoIP and P2P are undertaken by UDP. The UDP traffic becomes no more ignorable. Additionally, Comparing Table (3.a) and (3.b), it is easy to know that the average packet size of UDP is smaller than that of TCP. It is reasonable that some UDP applications for example VoIP tend to pack data with small packets. 
Table 3. Traffic in Transport Layer

\begin{tabular}{|c|c|c|c|c|c|c|}
\hline & \multicolumn{3}{|c|}{ In bytes (\%) } & \multicolumn{3}{c|}{ in packets (\%) } \\
\cline { 2 - 7 } & Inbound & Outbound & Bidirection & Inbound & Outbound & Bidirection \\
\hline TCP & 74.09 & 69.81 & 73.24 & 59.17 & 66.45 & 61.24 \\
\hline UDP & 25.83 & 30.06 & 26.67 & 40.44 & 32.95 & 38.31 \\
\hline ICMP & 0.06 & 0.13 & 0.07 & 0.36 & 0.60 & 0.43 \\
\hline Others & 0.02 & 0.00 & 0.02 & 0.03 & 0.00 & 0.02 \\
\hline
\end{tabular}

\subsection{Traffic in Application Layer}

We classify all applications into 7 application categories according to protocol definitions and traffic characteristics as table 4 [16].

Table 4. Application categories

\begin{tabular}{|l|c|}
\hline Application & Example Applications \\
\hline Protocols in RFCs & $\begin{array}{c}\text { HTTP, FTP, DNS, Telnet, SNMP, DHCP, } \\
\text { SMTP, POP }\end{array}$ \\
\hline P2P File Sharing & $\begin{array}{c}\text { Bittorrent, eDonkey, Gnutella, KAD, Fasttrack, } \\
\text { Freenet, Poco, Xunlei }\end{array}$ \\
\hline Instant Messenger & $\begin{array}{c}\text { ICQ, MSN Messenger, Napster, QQ, Skype, } \\
\text { Yahoo Messenger, Google Talk }\end{array}$ \\
\hline Games & $\begin{array}{c}\text { Balltefield 1942, Doom, Quake, Need for Speed, } \\
\text { Unreal, Xbox Live, Counter-Strike }\end{array}$ \\
\hline Streaming Media & RTSP, PNM, MMS, QuickTime \\
\hline VoIP & H.323, SIP, MEGACO, Google Talk, QQ \\
\hline Uncategorized & $\begin{array}{c}\text { VPN, Lotus Notes, Radius, pcAnywhere, } \\
\text { Oracle, CodeRed, Nimda, Worm }\end{array}$ \\
\hline
\end{tabular}

There are about 3.7 TB traffic for P2P file sharing applications, among them 2.6 TB for inbound and 1.1 TB for outbound. While for traditional applications such as HTTP and FTP, there are about 1.8 TB traffic in total and 1.7 TB for inbound and 0.1 TB for outbound. The traffic inbound and outbound are symmetrical for P2P file sharing due to hosts are playing as the roles of both severs and clients in P2P applications. But, there are few servers providing traditional services inside the MAN, so the traffic amount of outbound is much less than that of inbound for traditional applications. The traffic ratio of $\mathrm{P} 2 \mathrm{P}$ application is not so much traffic amount for $\mathrm{P} 2 \mathrm{P}$ applications as that reported by others in our measurement [14]. This phenomenon is unlikely caused by our traffic identification method MPI since the uncategorized traffics are impossible belonging to $\mathrm{P} 2 \mathrm{P}$ applications from their characteristics.

The packet size of the inbound traffic of traditional application is larger than that of outbound since the packets of inbound mostly are requested data while acknowledgements packets for outbound. Then, it is well understood that the traditional applications holds $19.56 \%$ in packets and $33.42 \%$ in bytes in inbound, but $16.70 \%$ in packets and $7.40 \%$ for outbound. The packet size is also very symmetrical for $\mathrm{P} 2 \mathrm{P}$ file sharing applications in both directions. The average packet size of uncategorized traffics is small and these traffics hold $32.59 \%$ in packets but only $19.99 \%$ in bytes. 
Table 5. Traffic in Applications Categories

\begin{tabular}{|l|c|l|l|c|c|c|}
\hline & \multicolumn{3}{|c|}{ In Bytes (\%) } & \multicolumn{3}{c|}{ In Packets (\%) } \\
\cline { 2 - 7 } & Inbound & Outbound & Bidirectional & Inbound & Outbound & Bidirectional \\
\hline Protocols in RFCs & 33.42 & 7.40 & 28.25 & 19.56 & 16.70 & 18.75 \\
\hline P2P File Sharing & 32.59 & 53.22 & 36.69 & 23.48 & 30.34 & 25.43 \\
\hline Instant Messenger & 0.30 & 0.22 & 0.29 & 0.48 & 0.59 & 0.51 \\
\hline Games & 3.13 & 2.73 & 3.05 & 7.97 & 4.71 & 7.04 \\
\hline Streaming Media & 7.46 & 5.21 & 7.01 & 5.29 & 5.91 & 5.47 \\
\hline VoIP & 5.03 & 3.53 & 4.73 & 11.71 & 6.41 & 10.21 \\
\hline Uncategorized & 18.07 & 27.71 & 19.99 & 31.50 & 35.34 & 32.59 \\
\hline
\end{tabular}

The traffic rates of all application categories are shown as Fig. 4. The time characteristic of traffic rate of $\mathrm{P} 2 \mathrm{P}$ applications is some different with others. In most time the P2P file sharing traffic is the largest part of all in bps, but the traffic rate of uncategorized applications is the largest one in pps (pakcets per second) since the packet size of the uncategorized traffic is smaller. The characteristic of period of every application category is very obvious and similar. But, the peak of traffic rate of $\mathrm{P} 2 \mathrm{P}$ delays back to others since more $\mathrm{P} 2 \mathrm{P}$ files are downloaded during sleeping time.
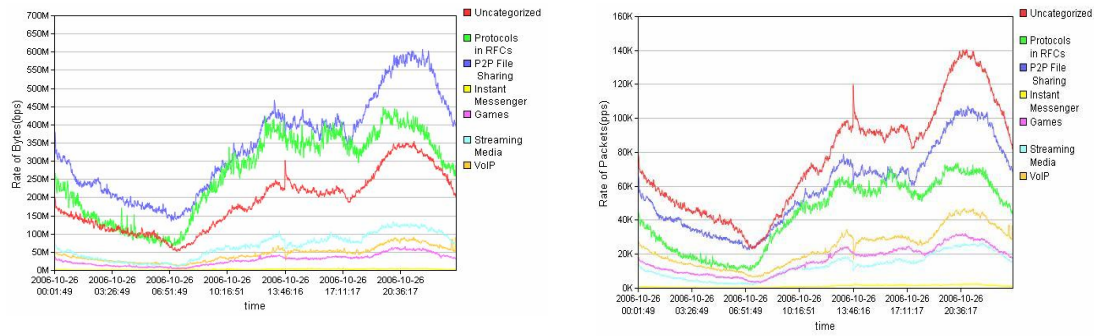

(B) IN pps

Fig. 4. Traffic Rate of Application Categories

A more detailed traffic amounts list of all applications is listed in Table 6. HTTP as an application, its traffic amount still is the largest both in byte and packet.

According to Table 6, there are about $9.39 \%$ packets for VoIP. The distribution of calling times is shown as Fig. 5. The $\mathrm{x}$ axis 1 to 14 denote less than 1, 1-2, 2-3, 3-4, 4$5,5-6,6-7,7-8,8-9,9-10,10-20,20-30,30-60$ and more than 60 minutes. There are about $5 \%$ callings more than 10 minutes and about $69.93 \%$ callings in 1 minute. One of the reasons of many sessions of VoIP less than 1 minute is the failure to set up the connection.

\subsection{IP Pair and Flow}

IP pair and flow are also checked in this paper to describe the behaviors of hosts and sessions.

The concurrent IP pairs are shown in Fig. 6. The maximum count of concurrent IP pairs is 196638, the minimum 35779 and average 10767. The time characteristic of concurrent IP pairs is similar to traffic rate as in Fig. 3. 
Table 6. Traffic in Applications

\begin{tabular}{|l|c|c|c|c|c|c|}
\hline Apps & Inbound & Outbound & Bidirectional & Inbound & Outbound & Bidirectional \\
\hline Http & 30.38 & 6.33 & 25.61 & 16.78 & 14.27 & 16.07 \\
\hline FTP Data & 0.69 & 0.0 & 0.55 & 0.42 & 0.01 & 0.30 \\
\hline SMTP & 0.0 & 0.02 & 0.01 & 0.01 & 0.01 & 0.01 \\
\hline POP3 & 0.03 & 0.0 & 0.02 & 0.01 & 0.01 & 0.01 \\
\hline DNS & 0.04 & 0.04 & 0.04 & 0.16 & 0.25 & 0.18 \\
\hline HTTPS & 0.62 & 0.65 & 0.63 & 0.86 & 1.06 & 0.92 \\
\hline Edonkey & 2.42 & 4.64 & 2.86 & 1.88 & 2.49 & 2.05 \\
\hline BitTorrent & 4.69 & 9.88 & 5.72 & 4.27 & 6.05 & 4.78 \\
\hline Poco & 1.01 & 1.68 & 1.14 & 1.15 & 1.05 & 1.12 \\
\hline lunlei & 4.66 & 9.69 & 5.66 & 3.83 & 5.23 & 4.23 \\
\hline P2P_Others & 19.78 & 27.32 & 21.28 & 12.33 & 15.51 & 13.23 \\
\hline MSN & 0.02 & 0.06 & 0.03 & 0.02 & 0.03 & 0.02 \\
\hline QQ & 0.28 & 0.15 & 0.26 & 0.46 & 0.56 & 0.49 \\
\hline Games & 3.13 & 2.73 & 3.05 & 7.97 & 4.71 & 7.04 \\
\hline PPLive & 1.44 & 1.84 & 1.52 & 1.80 & 1.89 & 1.83 \\
\hline PPstream & 1.80 & 2.71 & 1.98 & 1.60 & 2.16 & 1.76 \\
\hline Rtsp & 2.61 & 0.15 & 2.12 & 1.08 & $1.17 \%$ & 1.10 \\
\hline SM_Other & 0.69 & 0.38 & 0.63 & 0.36 & 0.26 & 0.33 \\
\hline Sip & 0.88 & 0.11 & 0.73 & 0.88 & 0.19 & 0.68 \\
\hline h323 & 0.09 & 0.17 & 0.10 & 0.09 & 0.13 & 0.10 \\
\hline VoIP_Others & 4.03 & 3.23 & 3.87 & 10.71 & 6.06 & 9.39 \\
\hline Total & 79.29 & 71.78 & 77.81 & 66.67 & 63.10 & 65.64 \\
\hline
\end{tabular}

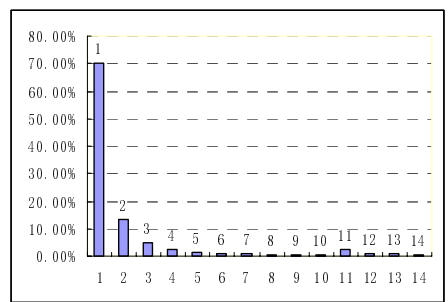

Fig. 5. Time Distribution of VoIP

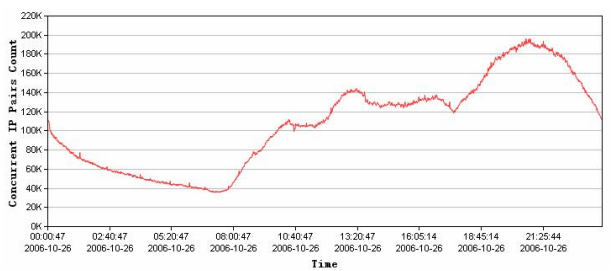

Fig. 6. Concurrent IP Pairs

The characteristic of concurrent flows as Fig. 7 is close to that of concurrent IP pairs as Fig.6. The maximum, minimum and average count of concurrent flows is 293591, 81663 and 163235. So, comparing with the count of concurrent IP pairs, there are only 1.5 concurrent flows for every IP pair averagely. 


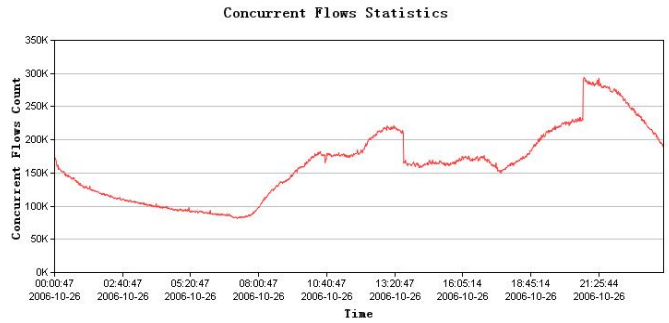

Fig. 7. Concurrent Flows

The rate of new added and closed flows is in direct proportion with the concurrent IP pairs shown in Fig. 8. The rates of new added and closed flows are almost same. The maximum, minimum and average rate of new added flows is 5967, 971, 3352 and that of closed is 6123,968 and 3356 respectively.

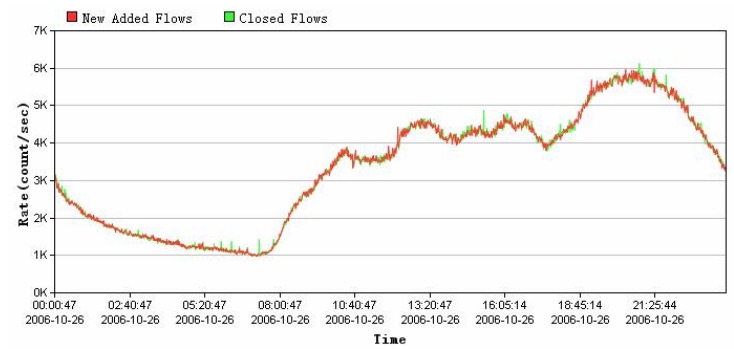

Fig. 8. The Rate of New Added and Closed flows

The $73.25 \%$ flows are less than $1 \mathrm{kB}$, about $19.56 \%$ from $1 \mathrm{~KB}$ to $10 \mathrm{~KB}, 5.58 \%$ from $20 \mathrm{~KB}$ to $160 \mathrm{~KB}, 0.61 \%$ more than $1 \mathrm{MB}$ and about $0.08 \%$ flows more than 5 $\mathrm{MB}$ as Fig. 9.a. There are $80 \%$ flows are less than 10 packets and $0.59 \%$ flows are more than 1000 packets. This result is also some different with previous research of Brownlee et al. [21] which shows for Web streams $87 \%$ under $1 \mathrm{kB}, 8 \%$ between 1 and $10 \mathrm{kB}$ and $4.8 \%$ between 10 and $100 \mathrm{kB}$. As to Non-web streams there is $89 \%$ under $1 \mathrm{kB}, 7 \%$ between 1 and $10 \mathrm{kB}$ and $1.5 \%$ between 10 and $100 \mathrm{kB}$. Comparing with previous result, the flows with lager workload increase with the possible reason the web pages tend to get larger with pictures and the flows of P2P and VoIP increase.

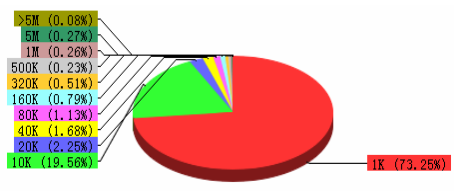

(A) BYTES

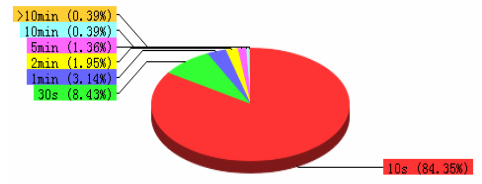

(B) LASTING TIME

Fig. 9. Distribution of Flows 
The sessions of $84.35 \%$ flows are less than 10 seconds, $8.43 \%$ lasting 10 seconds to 30 seconds, $3.14 \%$ lasting 30 seconds to 60 seconds, $3.7 \%$ lasting 1 minute to 10 minutes and only $0.39 \%$ flows lasting more than 10 minutes.

\section{Related Work}

Traffic metrics are essential for network research, planning and operating. Due to its fundamental nature, the research of traffic measurement and modeling has maintained continuous interest.

There are lots of works on the methodologies of traffic identification recent years since there are more and more applications using dynamic protocol ports bringing great challenge to it $[13,14,16]$. To address the inaccurate classification of network traffic just by a simple inspection of protocol port number used by flows, Andrew W. Moore and Konstantina Papagiannaki devise a contect-based classification scheme utilizing the full packet payload [13]. But, this methodology is not automated and may require human intervention. Consequently, the methodology is not suitable for realtime measurement system. So, it is difficult to get long term traffic metrics with detailed breakdown with this method. In this paper we present these metrics with on line measurement thanks to the traffic identification ability of MPI.

There are also lots of works on traffic models and characteristics for WAN or LAN, for packet-level and flow-level [5, 6, 10, 19], or applications. These works analyzed one of features and characterize with the theory of stochastic processes. Ruoming [9] presents a broad overview of internal enterprise traffic recorded at a medium sized site based on packet traces which describes some characteristic of traditional applications such as HTTP, Email, DNS and other enterprise-only applications. But, the traffic composing is highly different between different enterprises. That decreases the significance for measurement on enterprise traffic.

More related works are presented in paper [19, 20, 21, 22]. The traffic characteristics have been changed greatly with new applications appearing as we point out in previous sections. The newest traffic measurement from Fraleigh is published in 2003 [22]. In their experiment, a packet captured and analysis system is developed with 10 terabyte storage area network for packets storage and with a computing cluster for analyzing the trace. The results include traffic workload, TCP flow round-trip time, out of sequence packet rates and packet delay. The traffic characteristics for example traffic breakdown in different protocol layers look extraordinary different with our result, even for traffic rate which is more smooth than that in their result. It goes without saying for these changes due to applications and access networks also change greatly. So, the traffic should be measured and analyzed with more powerful online measurement system and more accurate traffic identification methods. It is impossible to measure traffic based on packet trace for high speed network today as previous works [22, 23].

\section{Conclusions}

Metro area network (MAN) as PoPs of Internet connecting millions of users into backbone plays key role in Internet. Network traffic measurements can provide 
essential data for network research and operation. Under the characteristic of MAN traffic is very important for performance traffic engineering to improve performance in MAN. The major contribution of this paper is to provide a broad view of MAN network traffic from packet, host and flow. We present a detailed breakdown of traffic from data link layer to application layer based on measurement the export link of MAN on line. To the best of our knowledge, this is the first work introducing these so broad metrics with on line and long term measurement which can provide first hand metrics about traffic of MAN. We find the characteristics of traffic have changed greatly from previous measurements. Also, we explain the reasons for these changes.

Obviously, our investigation is only the first step on the research of traffic characteristic. We are divided ascend, descend, peak, and bottom phase according to the traffic rate and discussing the traffic characteristic during different phases. More profound traffic models on flows and applications will also be researched in future work.

\section{Acknowledgment}

This research is funded by National Natural Science Foundation of China with grant no. 60403031 and 90604015 and the Foundation of French-Chinese for Sciences and their Applications (FFCSA 2005-2006). This paper is also supported by France Telecom R\&D with grant no. 46135216 and China Mobile. Thanks for others efforts on development the measurement system, especially thanks for Liang SHUAI, Zhenbao ZHOU, Shuangguang WEN and Da PENG.

\section{References}

1. Ibrahim Hamdy, A., Nossier Bahnasy, M., Darwish Mohamed, G.: Billing system for Internet Service Provider (ISP), IEEE MELECON 2002: Mediterranean electrotechnical conference (2002)

2. Mortier, R.: Internet Traffic Engineering, Technical Report of University of Cambridge, UCAM_CL_TR_532 (2002)

3. Mai, J., Chuah, C.-N., Sridharan, A., Ye, T., Zang, H.: Is Sampled Data Sufficient for Anomaly Detection? In: IMC 2006. Rio de Janeiro, Brazil (October 2006)

4. Duffield, N.G., Lund, C.: Predicting Resource Usage and Estimation Accuracy in an IP Flow Measurement Collection Infrastructure. ACM SIGCOMM Internet Measurement Conference 2003, Miami Beach, Fl, (October 27-29, 2003)

5. Leland, W., Taqqu, M., Willinger, W., Wilson, D.: On the self-similar nature of Ethernet traffic. In: Proceedings of SIGCOMM '93, pp. 183-193 (September 1993)

6. Shakkottai, S., Brownlee, N., Claffy, K.C.: Study of Burstiness in TCP Flows, PAM ( 2005)

7. Ramon Caceres, Measurement of wide area internet traffic. Technical report of UC Berkeley (1989) http://www.kiskeya.net/ramon/work/pubs/ucb89.pdf

8. Leland, W.E., Wilson, D.V.: High time-resolution measurement and analysis of LAN traffic: Implications for LAN interconnection. In: Proceeedings of IEEE Infocomm '91, pp. 1360-1366, Bal Harbour, FL (1991)

9. Pang, R., Allman, M., Bennett, M., Lee, J., Paxson, V., Tierney, B.: A First Look at Modern Enterprise Traffic. In: Proceeding of Internet Measurement Conference 2005 (2005) 
10. Mah, B.: An empirical model of HTTP network traffic. In: Proceedings of INFOCOM 97 (April 1997)

11. Fraleigh, C., Moon, S., Lyles, B., Cotton, C., Khan, M., Moll, D., Rockell, R., Seely, T., Diot, C.: Packet-Level Traffic Measurements from the Sprint IP Backbone. In: IEEE Network (2003)

12. Jaiswal, S., Iannaccone, G., Diot, C., Kurose, J., Towsley, D.: Measurement and Classification of Out-of-Sequence Packets in a Tier-1 IP Backbone. In: IEEE Infocom. San Francisco (March 2003)

13. Moore, A., Papagiannaki, K.: Toward the Accurate Identification of Network Applications. In: Proceedings of PAM (2005)

14. Karagiannis, T., Broido, A., Brownlee, N., Claffy, Kc., Faloutsos, M.: Is P2P Dying or Just Hiding. In: Proceeding of IEEE Globecom, Dallas (2004)

15. Xie, G., Yang, J., Issarny, V., Conte, A.: An Accurate and Efficient 3-Phases Measurement Method for IP Traffic Flow on High Speed Link (to Submitted)

16. Xie, G., Zhang, G., Yang, J., Issarny, V., Conte, A.: Accurate Online Traffic Classification with Multi-phases Identification Methodology (to Submitted)

17. Duffield, N.G., Lund, C., Thorup, M.: Learn more, sample less: control of volume and variance in network measurement. IEEE Transactions in Information Theory 51(5), 17561775 (2005)

18. Barakat, C., Thiran, P., Iannaccone, G., Diot, C., Owezarski, P.: Modeling Internet Backbone Traffic at the Flow Level. In: IEEE Transactions on Signal Processing (Special Issue on Networking) (August 2003)

19. Thompson, K., Miller, G.J., Wilder, R.: Wide-area Internet traffic patterns and characteristics. IEEE Network, 11(6) (November 1997)

20. McCreary, S., Claffy, K.: Trends in wide area IP traffic patterns - A view from Ames Internet Exchange, ITC Specialist Seminar /AIX0005/ (2000) http://www.caida.org/publications/papers/

21. Brownlee, N., Claffy, K.C.: Internet stream size distributions. In: Proc. ACM SIGCOMM, pp.282-283 (2002)

22. Fraleigh, C., Moon, S., Lyles, B., Cotton, C., Khan, M., Moll, D., Rockell, R., Seely, T., Diot, C.: Packet-level traffic measurements from the Sprint IP backbone. IEEE Network 17(6), 6-16 (2003)

23. Azzouna, N.B., Guillemin, F.: Impact of peer-to-peer applications on wide area network traffic: an experimental approach. GLOBECOM '04. IEEE. 29 Nov.-3 Dec. 2004 3, 1544 1548 (2004) 